\title{
Idiopathic mesenteric phlebosclerosis associated with long-term use of Chinese herbal medicine
}

\author{
Hsing-Jung Yeh ${ }^{1,2}$, Pao-Ying Lin ${ }^{1,2}$, Wei-Yu Kao ${ }^{1,2}$, Ching-Huei Kun ${ }^{3}$, Chun-Chao Chang ${ }^{1,2}$ \\ ${ }^{1}$ Division of Gastroenterology and Hepatology, Department of Internal Medicine, Taipei Medical University Hospital, Taipei, Taiwan \\ ${ }^{2}$ Division of Gastroenterology and Hepatology, Department of Internal Medicine, Taipei Medical University School of Medicine, Taipei, Taiwan \\ ${ }^{3}$ Department of Medical Imaging, Taipei Medical University Hospital, Taipei, Taiwan
}

Cite this article as: Yeh HJ, Lin PY, Kao WY, Kun $\mathrm{CH}$, Chang CC. Idiopathic mesenteric phlebosclerosis associated with long-term use of Chinese herbal medicine. Turk J Gastroenterol 2018; 29: 140-2.

To the editors,

Our 60 year-old female menopausal patient took a Chinese herbal medicine from a herbal pharmacy. Because she had pyrosis, herbal pharmacist added an extract of Gardenia jasminoides to her prescription; she was not evaluated or diagnosed by any doctor. According to her statement, she used this medicine for more than eight years.

She suffered from intermittent dull abdominal pain and bloody stools for six months. Sometimes, she suffered from constipation. She lost approximately $6 \mathrm{~kg}$ of her body weight in three months. She visited a health evalua- tion center and was transferred to the outpatient department of our hospital. She did not have any special medical history, except for a prolapsed mitral valve. Her family had no history of colorectal cancer or colitis. She had mild pale conjunctivae and her laboratory data revealed mild anemia. She had abdominal distension and right-sided abdominal tenderness. Colonoscopy revealed that colonic ulcers and bluish discoloration of the colonic mucosa that was noted from the cecum to the proximal descending colon (Figure 1a). It is easy to cause colonic mucosal bleeding by touching with forceps (Figure 1b). Abdominal computed tomography revealed a thickened bowel wall from the ascending colon to the proximal de-
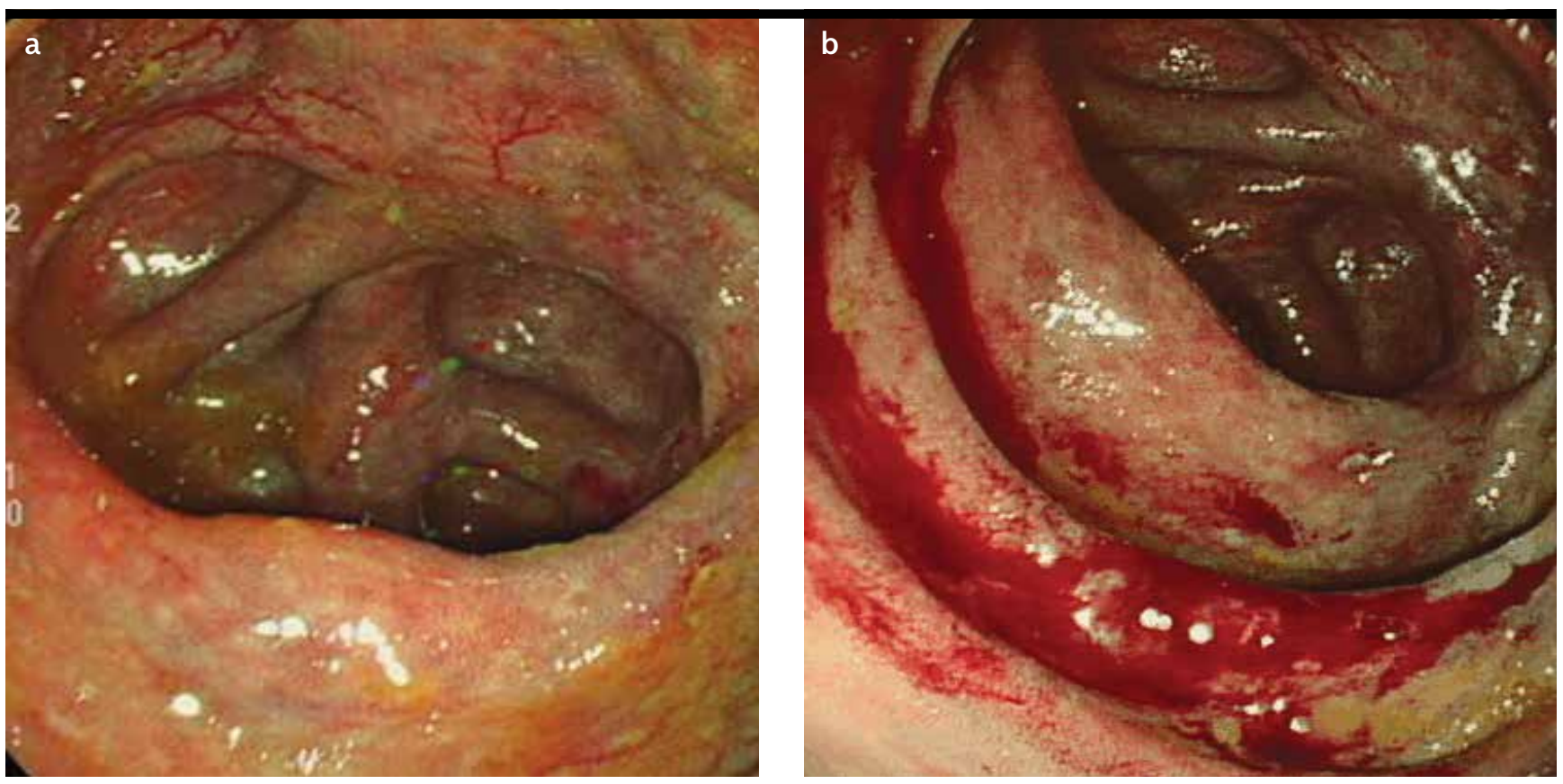

Figure 1. a, b. Endoscopic images showing dark purple mucosa at the cecum and ascending colon (a). The mucosa was easily touch bleeding (b).

Address for Correspondence: Chun-Chao Chang E-mail: Chunchao@tmu.edu.tw Received: March 21, 2017 Accepted: September 07, 2017

(C) Copyright 2018 by The Turkish Society of Gastroenterology · Available online at www.turkjgastroenterol.org DOI: $10.5152 /$ tjg.2018.17072 

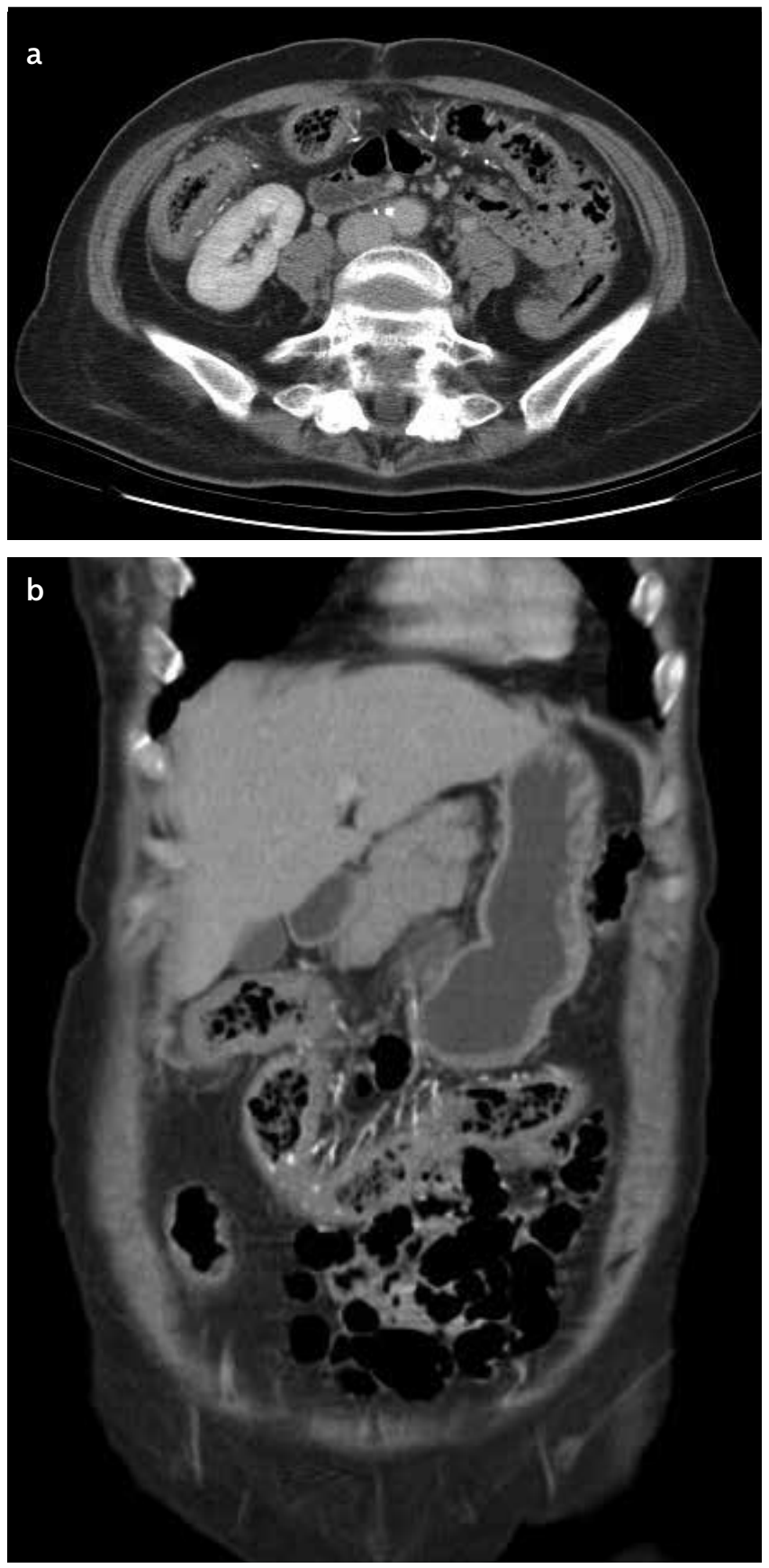

Figure 2. a, b. Axial (a) and coronal (b) computed tomography images showing thickened bowel walls at the proximal descending colon, transverse colon, ascending colon, and ileocecal region with serpiginous calcification at the mesenteric vessels

scending colon and a calcified mesenteric vessels (Figure $2 a, b)$. Random biopsies were obtained from the colonic mucosa. The pathology result revealed some lymphoplasma cell infiltration in the edematous lamina propria. No crypt abscess or granuloma was observed.
Thus, this patient was diagnosed as having idiopathic mesenteric phlebosclerosis (IMP). She stopped using the herbal medicine she had taken from the herbal pharmacy. Because she was not suitable for undergoing surgery, we give her symptomatic treatment. Finally, her intermittent abdominal pain and bloody stools was improved after treatment and she was regular followed up was advised.

IMP was first reported by Koyama et al. (1) in 1991. It was named by Iwashita et al. (2) in 2003. It is characterized by thickening of the wall of the right hemi-colon and calcification of the corresponding mesenteric veins. Because the patient often had intermittent abdominal pain and bloody stools, this disease should be made differential diagnosis from Crohn's disease, ulcerative colitis, colorectal cancer, diverticulitis, ischemic bowel disease and amyloidosis, etc. Colonoscopy images mostly revealed an edematous purple mucosa and ulcers, which should be differentiated from other diseases. IMP is diagnosed based on its characteristic radiographic findings of threadlike calcifications in the mesenteric veins (3). CT is the most useful diagnostic tool for evaluating the severity of mesenteric vein calcification (3). We ever reported the case of a female patient suffering from IMP who had an obstruction and who was diagnosed by CT (4).

In 2012, Hiramatsu et al. (5) reported IMP patients often have a history of the long-term use of a Chinese herbal medicine. These Chinese herbal medicines usually included Sansisi (G. jasminoides). An extract of G. jasminoides can be used as a herbal medicine; this has been used for more 3000 years. . Geniposide is the main component of G. jasminoides which is usually prescribed for women in Taiwan. Therefore, patients who took herbal medicines containing an extract of $\mathrm{G}$. jasminoides might absorb a high amount of geniposide.

Geniposide could be transferred to genipin. Genipin affects right hemi-colonic bacteria flora and vascular system. The purple mucosa might be related to geniposide and the change of enterobacterial flora. Because genipin damages colonic mucosa, these patients have mesenteric phlebosclerosis, purple colonic mucosa, and colonic ulcers. No effective medicine for IMP, except stop using herbal medicines to avoid disease progression. Patients should not take the same herbal medicine for a long time without being evaluated by a doctor, particularly a medicine containing a $\mathrm{G}$. jasminoides extract.

Peer-review: Externally peer-reviewed. 
Author contributions: Concept - H.J.Y., C.C.C.; Design - H.J.Y.; Supervision - C.C.C.; Materials - C.C.C.; Data Collection and/or Processing - C.H.K.; Literature Search - H.J.Y.; Writing - H.J.Y.; Critical Reviews - C.C.C.

Conflict of Interest: No conflict of interest was declared by the authors.

Financial Disclosure: The authors declared that this study has received no financial support.

\section{REFERENCES}

1. Koyama N, Koyama H, Hanajima T, et al. Chronic ischemic colitis causing stenosis, report of a case. I to Cho (Stomach and Intestine) 1991; 26: 455-60.
2. Iwashita A, Yao T, Schlemper RJ, et al. Mesenteric phlebosclerosis : a new disease entity causing ischemic colitis. Dis Colon Rectum 2003; 46; 209-20. [CrossRef]

3. Yen TS, Liu CA, Chiu NC, Chiou YY, Chou YH, Chang CY. Relationship between severity of venous calcifications and symptoms of phlebosclerotic colitis. World J Gastroenterol 2015 July 14; 21: 8148-55. [CrossRef]

4. Lin PY, Chai CY, Chang CC. Recurrent abdominal pain in a 55-year-old woman. Diagnosis: Idiopathic mesenteric phlebosclerosis. Gastroenterology 2011; 141: 36-404. [CrossRef]

5. Hiramatsu K, Sakata H, Horita Y, et al. Mesenteric phlebosclerosis associated with long term oral intake of geniposide, an ingredient of herbal medicine. Aliment Pharmacol Ther 2012; 36: 575-86. [CrossRef] 